\title{
Diagnostic Neuroimaging in Headache Patients: A Systematic Review and Meta-Analysis
}

\author{
Ye Eun Jang ${ }^{1}$, Eun Young $\mathrm{Cho}^{2}$, Hee Yea Choi ${ }^{1}$, Sun Mi Kim ${ }^{3}$, and Hye Youn Park ${ }^{1}$ \\ ${ }^{1}$ Department of Psychiatry, Seoul National University of Bundang Hospital, Seongnam, Republic of Korea \\ ${ }^{2}$ Department of Biostatistics, Korea University Graduate School, Seoul, Republic of Korea \\ ${ }^{3}$ Department of Psychiatry, Chung-Ang University Medical Center, Seoul, Republic of Korea
}

\begin{abstract}
Objective Neuroimaging in headache patients identifies clinically significant neurological abnormalities and plays an important role in excluding secondary headache diagnoses. We performed a systematic review and meta-analysis of the existing guidelines and studies surrounding neuroimaging in headache patients.

Methods The research question involved determining the prevalence of detecting clinically significant neurological abnormalities using neuroimaging in patients suspected of primary headache. Searches of the PubMed and Embase databases were conducted on English-language studies published from 1991 to 2016, and the reference lists of the retrieved articles were also checked manually. All headache subtypes and patients aged $\geq 15$ years were included in the analysis.

Results Ten studies met the selection criteria. The pooled prevalence of detecting clinically significant abnormalities in the neuroimaging of headache patients was $8.86 \%$ (95\% confidence interval: $5.12-15.33 \%)$. Subsequently, diverse subgroup analyses were performed based on the detection method, headache type, study type, study region, age group, and disease type.

Conclusion The present findings indicate that limited neuroimaging methods should be carefully considered for headache diagnostic purposes when there are red flag symptoms. Limitations and suggested directions for future studies on neuroimaging in headache patients are described.

Psychiatry Investig 2019;16(6):407-417
\end{abstract}

Key Words Headache, Neuroimaging, X-ray computed tomography, Magnetic resonance imaging.

\section{INTRODUCTION}

Headache is a common and disabling disease around the world. ${ }^{1}$ The most important methods for diagnosing headaches and determining the headache type are neurological examinations and a obtaining detailed history of the patient's symptoms. ${ }^{2}$ The screening of secondary headaches is also performed by identifying high-risk factors of sinister pathologies, such as new outbreaks, or changes in the symptoms of headache patients older than 50 years. ${ }^{3}$ The International Classification of Headache Disorders, 3rd edition (ICHD-3) comprises two main categories: primary and secondary headache disorders. ${ }^{1}$

Received: November 23, 2018 Revised: April 1, 2019

Accepted: April 11, 2019

$\triangle$ Correspondence: Hye Youn Park, MD

Department of Psychiatry, Seoul National University Bundang Hospital, 82 Gumi-ro 173beon-gil, Bundang-gu, Seongnam 13620, Republic of Korea Tel: +82-31-787-7441, Fax: +82-31-787-4058, E-mail: quteray@naver.com

(c) This is an Open Access article distributed under the terms of the Creative Commons Attribution Non-Commercial License (https://creativecommons.org/licenses/bync/4.0) which permits unrestricted non-commercial use, distribution, and reproduction in any medium, provided the original work is properly cited.
Primary headache disorders exhibit a benign non-organic etiology such as migraines, tension-type headaches, and cluster headaches. Secondary headache disorders are caused by organic diseases such as stroke, head and/or neck trauma, brain tumors, cranial cervical vascular disorders, and non-vascular intracranial disorders. ${ }^{4}$

According to the UK National Clinical Guideline Centre's guidelines, ${ }^{3}$ the traditional method of diagnosing primary headaches does not require neuroimaging, which should be avoided if unlikely to change the management of care or reveal any abnormalities. There are several reasons for this selective use of neuroimaging in headache patients: 1 ) it is not cost effective; 5 , 2) neuroimaging can cause the patient anxiety, which may impact the results and promote clinical uncertainty, ${ }^{7,8}$ it has the potential risk of providing false-positive results, and, thus, further evaluations that would otherwise be unnecessary may be required, and neuroimaging can detect clinically insignificant abnormalities, such as sinusitis, that do not impact the pathology behind headache and, therefore, would not change the clinical therapeutic approach; and 3) 
the overuse of some neuroimaging tools may cause severe side effects in patients. ${ }^{9} 10$ For example, although computed tomography (CT) and CT angiography (CTA) are convenient neuroimaging tools, they can cause allergic reactions and expose the patient to radiation.

Neuroimaging should be performed, however, on those suspected of an underlying disorder based on the presence of additional symptoms and signs that do not fit the clinical diagnosis of primary headache (e.g., atypical headache patterns, a history of seizures, and/or focal neurological symptoms or signs). Clinical guideline pertaining to neurophysiological tests and neuroimaging procedures for non-acute headache recommend magnetic resonance imaging (MRI) for autonomic nervous headache. ${ }^{2}$ An international guideline published in 2013 reported brain imaging to be beneficial for patients aged $>50$ years with new-onset headaches. ${ }^{11} \mathrm{MRI}$ is more sensitive than CT in screening for secondary headache, but a CT brain scan should be obtained as soon as possible when subarachnoid hemorrhage (SAH) is suspected. ${ }^{12}$ According to a systematic literature review, ${ }^{13}$ neuroimaging tests should be considered for patients with cluster headaches, acute severe headache, human immunodeficiency virus (HIV), or changes in consciousness and cognitive function. Furthermore, some recommend neuroimaging for headaches accompanied by nausea or vomiting and those worsened by Valsalva maneuver, such as coughing during thunderclap headaches. ${ }^{14}$ In addition, neuroimaging evaluations, such as CT, CTA, MRI, and magnetic resonance angiography (MRA), may be necessary for a differential diagnosis in the case of pregnancy, postpartum headache, and headaches aggravated by sexual activity or exercise. ${ }^{15}$

Although previous studies and literature reviews have provided consistent conclusions on when neuroimaging for headaches should be implemented, clear evidence supporting these recommendations are lacking because most studies have been case series or expert opinions, and there is a paucity of data from randomized clinical trials. ${ }^{2,11,12,15}$ Frishberg et al. ${ }^{16}$ conducted a systematic review and meta-analysis on non-acute headache patients diagnosed by CT or MRI. Even though they found significant intracranial abnormalities in patients with migraine headaches $(\sim 0.2 \%)$, there was insufficient data for patients with tension-type and unspecified headache types. Tsushima and Endo ${ }^{17}$ evaluated the ability of MRI to detect neurological abnormalities in chronic or recurrent headache patients and reported detection rates of $2.1 \%$ in unspecified headache patients, $0.52 \%$ in migraine patients without complications, and $26.3 \%$ in migraine patients with complications. Alons et al. ${ }^{9}$ conducted a systematic review on the utility of CT in patients with acute headache. The prevalence of vascular abnormalities in acute headache patients was $7.4 \%$, and $1.6 \%$ of the abnormalities were presumed to be the cause of the headache. Because these meta-analyses focused on limited ranges of headache types and neuroimaging tools, further analyses of the various headache types and neuroimaging tools evaluated in more recent articles are required.

Therefore, the present report will provide a systematic literature review and meta-analysis of recent studies; all headache subtypes were included given that the purpose of this study was to aid in making appropriate differential diagnoses. The research question was formulated based on the PICO (patients, intervention used, comparison and outcome) model. ${ }^{18}$

Research Question: In patients with suspected primary headache who underwent neuroimaging evaluations to identify the cause of headache, what is the prevalence of detecting a clinically important brain structure abnormality such as cancer, deformity, hemorrhage, or infarction by neuroimaging?

In addition, various subgroup meta-analyses were conducted according to the detection method, headache type, study type, study region, age group, and disease type to identify the sources of group heterogeneity and provide specific information on neuroimaging of headache patients.

\section{METHODS}

\section{Study search}

The systematic review was performed in accordance with the guidelines of the National Evidence-based Healthcare Collaborating Agency for systematic reviews. ${ }^{18}$ Computerassisted literature searches for relevant articles were conducted using the Seoul National University Medical Library. We performed a literature search of the PubMed and Embase databases up to January 2018 using the following key words: headache, cluster headache, migraine disorder, computed tomography angiography, CT, magnetic resonance imaging, and MRI. Specific search strategies reviewed by experts (Supplementary Table 1 in the online-only Data Supplement) were used to retrieve a total of 1,485 studies. The PubMed search using MeSH terms and the Embase search using Emtree terms yielded 354 and 1,131 articles, respectively. After excluding duplicates, 1,417 studies published from 1970 to January 2018 were included. Two authors (P.H.Y., J.Y.E) reviewed the titles and abstracts of each document using ENDNOTE X8 software and conducted individual reviews according to pre-set inclusion and exclusion criteria. The inclusion criteria were original reports involving CT/CTA/MRI/MRA neuroimaging of patients whose main symptom was headache. The exclusion criteria were as follows: language other than English; patient age $<15$ years; case studies, literature reviews, or meta-analyses; headache caused by trauma or injury; patients diagnosed with secondary headache; cost-effectiveness articles; and inability to distinguish among neuroimaging methods. 
When discrepancies occurred, consensus was achieved through group discussions. The reference lists of all retrieved studies were also checked manually. After reviewing the texts of the included studies, they were reassessed to narrow down the articles for inclusion in the meta-analysis. Studies selected independently by both authors using the aforementioned criteria were included in the final analysis. Ten studies corresponding to the research question were ultimately selected. A preferred reporting items for systematic reviews and metaanalyses (PRISMA) flowchart for the study selection process is shown in Figure 1.

\section{Risk of bias and data extraction}

The risk of bias was assessed using the Risk of Bias Tool developed by Hoy et al. ${ }^{19}$ used to evaluate the methodological quality of systematic reviews of non-randomized prevalence studies. This scale is based on a 'yes' or 'no' designation. Studies can be further classified as high risk if three or fewer items are designated as 'yes', moderate risk if four or five items are designated as yes, and low risk if six or more items are designated as 'yes'. Data pertaining to the following information was extracted: author/year, study type, sample size, numbers of males and females, age, sample source, country, headache subtype, number and percentage with abnormalities, type of detection tool, presence of contrast group, and risk-of-bias score (0-10). Risk-of-bias assessments and data extraction were performed by one author (J.Y.E) and supervised by the other (P.H.Y).

\section{Statistical analyses}

In this review, meta-analyses assessing the probabilities of detecting clinically significant abnormalities related to headache were performed; a clinically significant abnormality was considered to be an intracranial finding that may represent evidence of secondary headache or a need to change the therapeutic approach. Subsequently, subgroup meta-analyses of the clinically meaningful findings were performed. Meta-analyses were conducted using the meta package in $\mathrm{R}$ (version 3.5.0, The R Foundation, Vienna, Austria). To calculate the pooled proportion, the data were log transformed and evaluated by the Clopper-Pearson method to calculate 95\% confidence intervals (CIs). To estimate between-study heterogeneity, the $\mathrm{I}^{2}$ statistic was used. If the ratio of the actual variance $\left(\mathrm{I}^{2}\right)$ is $>50 \%$ and the significance of the homogeneity test $<0.10$, the heterogeneity of the effect size is considered substantial. ${ }^{20}$ Samples with high $\mathrm{I}^{2}$ values, indicating substantial heterogeneity, were included in the random-effects model. The random-effects meta-analysis model incorporates heterogeneity into the analysis, resulting in a wider CI and a more conservative claim of statistical significance. Subgroup analyses were performed according to the detection method (CT, CTA, MRI, and MRA), headache type (acute vs. non-acute), study type (prospective vs. retrospective), study region (Asia, Europe, or North America), age group ( $\leq 30,31-40,41-50,51-60$, and $>60$ years of age), and disease type (cerebrovascular disease, brain space-occupying lesions, infectious/inflammatory disease, and congenital human brain malformations). Although various types of disease were identified, they were classified

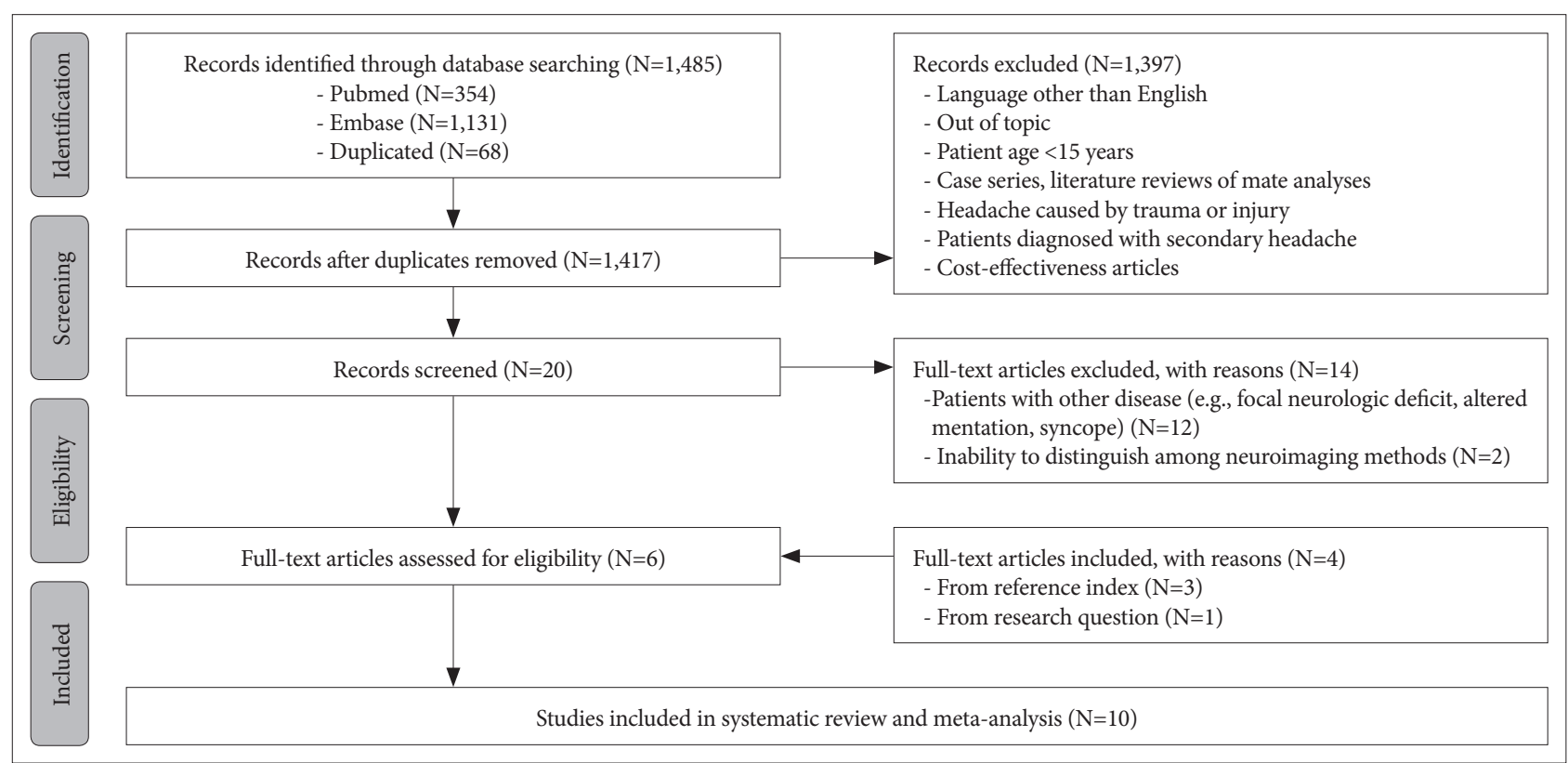

Figure 1. PRISMA flowchart of the study selection process. Preferred Reporting Items for Systematic reviews and Meta-Analysis (PRISMA) flow chart. 


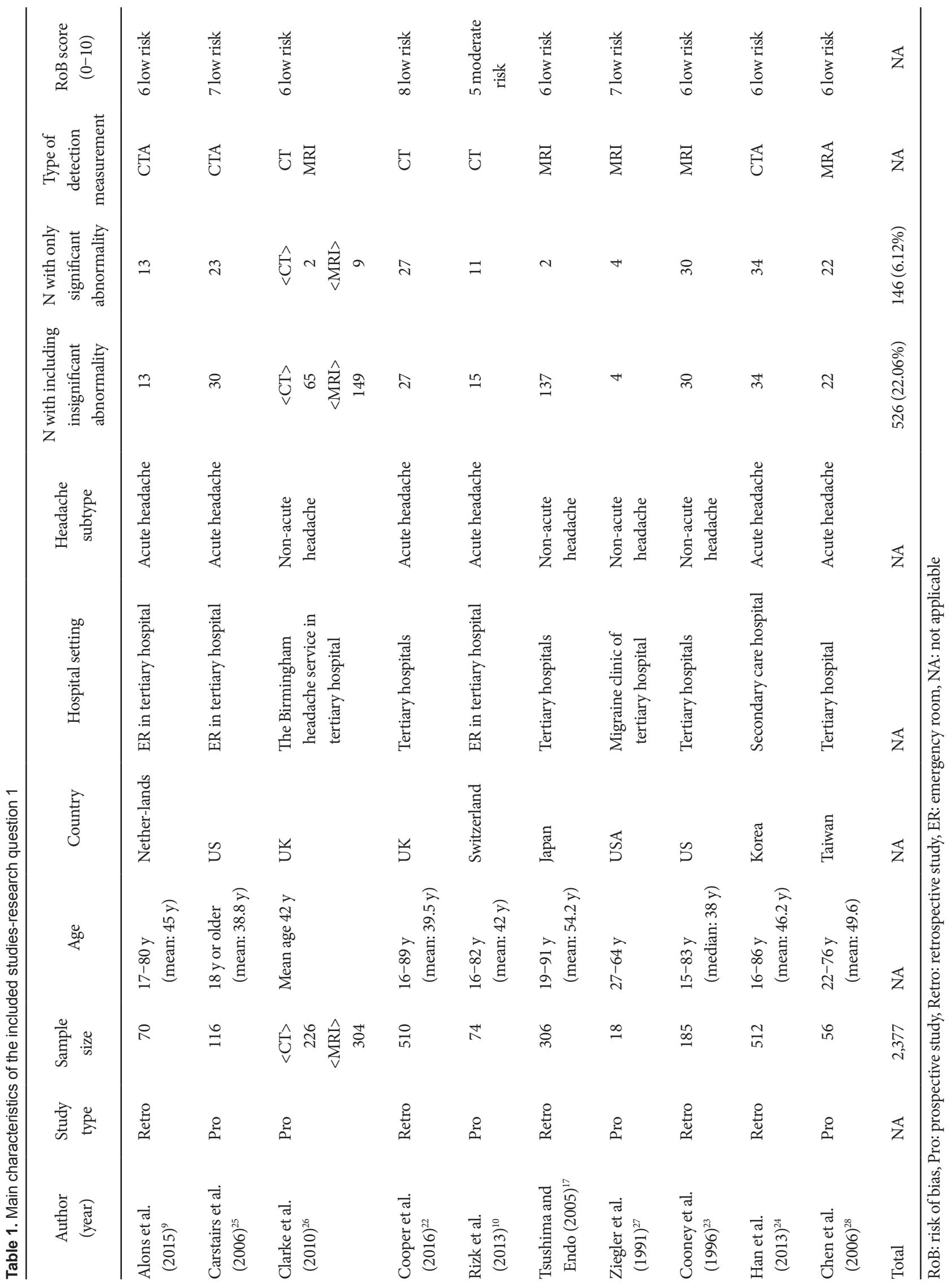


into the four aforementioned categories as follows: 1) cerebrovascular diseases included aneurysm/superior hypophyseal artery (SHA), infarcts, cerebral thrombosis, reversible cerebral vasoconstriction syndrome, cerebral ischemia, arteriovenous malformation, hypertensive emergency, temporal arteritis, chiari malformation, vasculitis, stroke, incidental cerebral aneurysm, subdural hemorrhage, moyamoya disease, arterial dissection, and segmental vasoconstriction; 2) brain space-occupying lesions included subdural hematoma, arachnoid cyst, pseudotumor cerebri, meningioma, metastatic disease, pituitary adenoma, neurosarcoidosis, hemangioma, bleed into glioblastoma, cavernoma, brain tumors, and pituitary macroadenoma; 3) infectious/inflammatory diseases included meningitis and sinusitis; and 4) congenital human brain malformations included Dandy-Waler syndrome. Publication bias was assessed by constructing a funnel plot in which each point denotes a single study. In the absence of bias, effect estimates of smaller studies are scattered at the lower end of the plot and those of larger studies clustered centrally toward the top, forming a symmetrical inverted funnel. ${ }^{21}$ Conversely, asymmetric distribution of the points in a funnel plot indicates the existence of publication bias.

\section{RESULTS}

\section{Summary of the included studies}

Ten studies ( $\mathrm{n}=2,377$ participants) were ultimately selected for the present meta-analysis. Of these 10 studies, five were retrospective analyses, ${ }^{9,17,22-24}$ and five were prospective anlayses. ${ }^{10,25-28}$ The studies were published between $1991^{27}$ and $2016^{22}$ and were conducted in the Netherlands, ${ }^{9}$ United States, ${ }^{23,25,27}$
United Kingdom, ${ }^{22,26}$ Switzerland, ${ }^{10}$ Japan, ${ }^{17}$ Korea, ${ }^{24}$ and Taiwan. ${ }^{28}$ Only headache patients aged 15 years or older were included. The headache subtypes in these studies included acute severe headache, ${ }^{9,10,22,24}$ non-acute headache, ${ }^{26}$ chronic or recurrent headache, ${ }^{17}$ migraine,${ }^{27,23}$ and thunderclap headache. ${ }^{25,28}$ The number of subjects included in each study ranged from $18^{27}$ to $510^{24}$ with a total number of 2,377 . Three studies used MRI, ${ }^{17,23,27}$ three used CTA, ${ }^{9,24,25}$ two used CT, ${ }^{10,22}$ one used MRA, ${ }^{28}$ and one used both MRI and CT. ${ }^{26}$ The characteristics of these 10 studies are presented in Table 1.

\section{Risk of bias (quality assessment)}

The risk of bias was assessed using the Risk-of-Bias Tool developed by Hoy et al. ${ }^{19}$ Of the 10 studies, nine were considered low risk, ${ }^{9,17,22-28}$ and one was of moderate risk. ${ }^{10}$ The risk-of-bias scores for each study are presented in Table 1.

\section{Meta-analyses}

Based on the results of the meta-analyses, the pooled prevalence of clinically significant findings was 8.86\% (95\% CI: $5.12-15.33 \%)$, and there was high inter-study heterogeneity $\left(\mathrm{Q}=147.12, \tau^{2}=0.7438, \mathrm{I}^{2}=93.2 \%, \mathrm{p}<0.01\right)$. A random-effects model was used because of the high $\mathrm{I}^{2}$ values, indicating substantial heterogeneity. Forest plots of the meta-analyses are presented in Figure 2.

\section{Subgroup meta-analyses}

Subgroup analyses were performed according to the detection method, headache type, study type, study region, age group, and disease type. The pooled prevalence rates of significant abnormalities were $4.98 \%$ as assessed by CT $(95 \%$
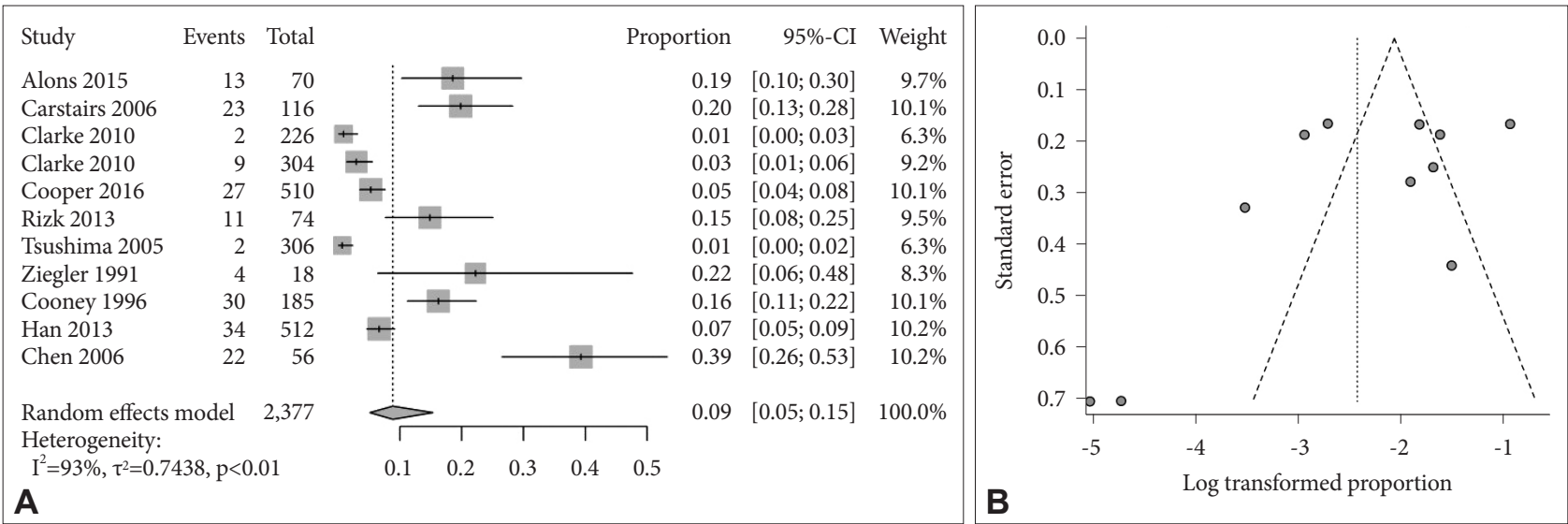

Figure 2. Forest plot and funnel plot for the Research Question. Forest plot (A) showing the prevalence of detecting clinically significant abnormalities. The events refer to cases in which abnormal findings were observed when neuroimaging was performed on the patients in each study. The total refers to the number of patients participating in each study, and the $x$-axis represents the confidence interval. When the confidence interval does not include zero, the incidence is not zero. Publication bias was tested using funnel plots. Funnel plot (B) showing the log-transformed proportion of detecting clinically significant abnormalities. To calculate the pooled proportion, the data were log-transformed and evaluated by the Clopper-Pearson method to calculate 95\% confidence intervals (Cls). Asymmetrical points indicate the presence of publication bias. 
CI: $1.67-14.88 \%), 13.33 \%$ as assessed by CTA (95\% CI: $6.24-$ 28.49\%), 39.29\% as assessed by MRA (95\% CI: $28.37-54.41 \%$ ), and $5.70 \%$ as assessed by MRI (95\% CI: $1.63-20.01 \%)$. There was also high inter-study heterogeneity $\left(\mathrm{Q}=18.05-39.96, \tau^{2}=\right.$ $\left.0.4090-1.4514, \mathrm{I}^{2}=88.9-92.5 \%, \mathrm{p}<0.01\right)$. The pooled prevalence rates of significant abnormalities associated with acute and non-acute headache were $13.98 \%$ (95\% CI: 7.14-27.39\%) and $4.06 \%$ (95\% CI: $1.20-13.77 \%$ ), respectively, with high inter-study heterogeneity $\left(\mathrm{Q}=52.23-90.56, \tau^{2}=0.6625-1.6982\right.$, $\left.\mathrm{I}^{2}=92.3-94.5 \%, \mathrm{p}<0.01\right)$. The pooled prevalence rates of significant abnormalities in the prospective and retrospective studies were $10.64 \%$ (95\% CI: 4.60-24.63\%) and 7.31\% (95\% CI: 3.77-14.18\%), respectively, with high inter-study heterogeneity $\left(\mathrm{Q}=46.02-71.08, \tau^{2}=0.4790-0.9630, \mathrm{I}^{2}=91.3-93.0 \%\right.$, $\mathrm{p}<0.01)$. The pooled prevalence rates of significant abnormalities were $18.03 \%$ for studies conducted in North America (95\% CI: 14.26-22.81\%), 6.43\% for studies conducted in Asia (95\% CI: $1.25-32.95 \%$ ), and 5.98\% for studies conduct- ed in Europe (95\% CI: 2.66-13.46\%), with high betweenstudy heterogeneity $\left(\mathrm{Q}=0.89-77.52, \tau^{2}=0.0-1.9233, \mathrm{I}^{2}=0.0^{-}\right.$ $97.4 \%, \mathrm{p}<0.01)$. The pooled prevalence rates of significant abnormalities were $5.73 \%$ in the $\leq 30$ group (95\% CI: $2.72-$ $12.07 \%$ ), $7.34 \%$ in the $31-40$ group (95\% CI: $4.21-12.78 \%$ ), $14.60 \%$ in the $41-50$ group (95\% CI: $3.67-58.03 \%$ ), $20.29 \%$ in the 51-60 group (95\% CI: $5.86-70.20 \%$ ), and $26.93 \%$ in the $>60$ group ( $95 \% \mathrm{CI}: 8.69-83.52 \%$ ), with high betweenstudy heterogeneity $\left(\mathrm{Q}=0.54-28.73, \tau^{2}=0.0-1.3569, \mathrm{I}^{2}=0.0^{-}\right.$ $93.0 \%, \mathrm{p}<0.01)$. Lastly, the pooled prevalence rates for significant abnormalities were $4.31 \%$ for cerebrovascular disease (95\% CI: 2.01-9.24\%), 1.35\% for brain space-occupying lesions (95\% CI: $0.59-3.11 \%$ ), $0.72 \%$ for infectious/inflammatory disease (95\% CI: $0.20-2.57 \%$ ), and $0.36 \%$ for congenital human brain malformations (95\% CI: 0.16-0.81\%), with high between-study heterogeneity $\left(\mathrm{Q}=5.12-137.26, \tau^{2}=0.0-1.2185\right.$, $\left.\mathrm{I}^{2}=0.0-92.7 \%, \mathrm{p}<0.01\right)$. Forest plots obtained from the subgroup analyses are presented in Figure 3.

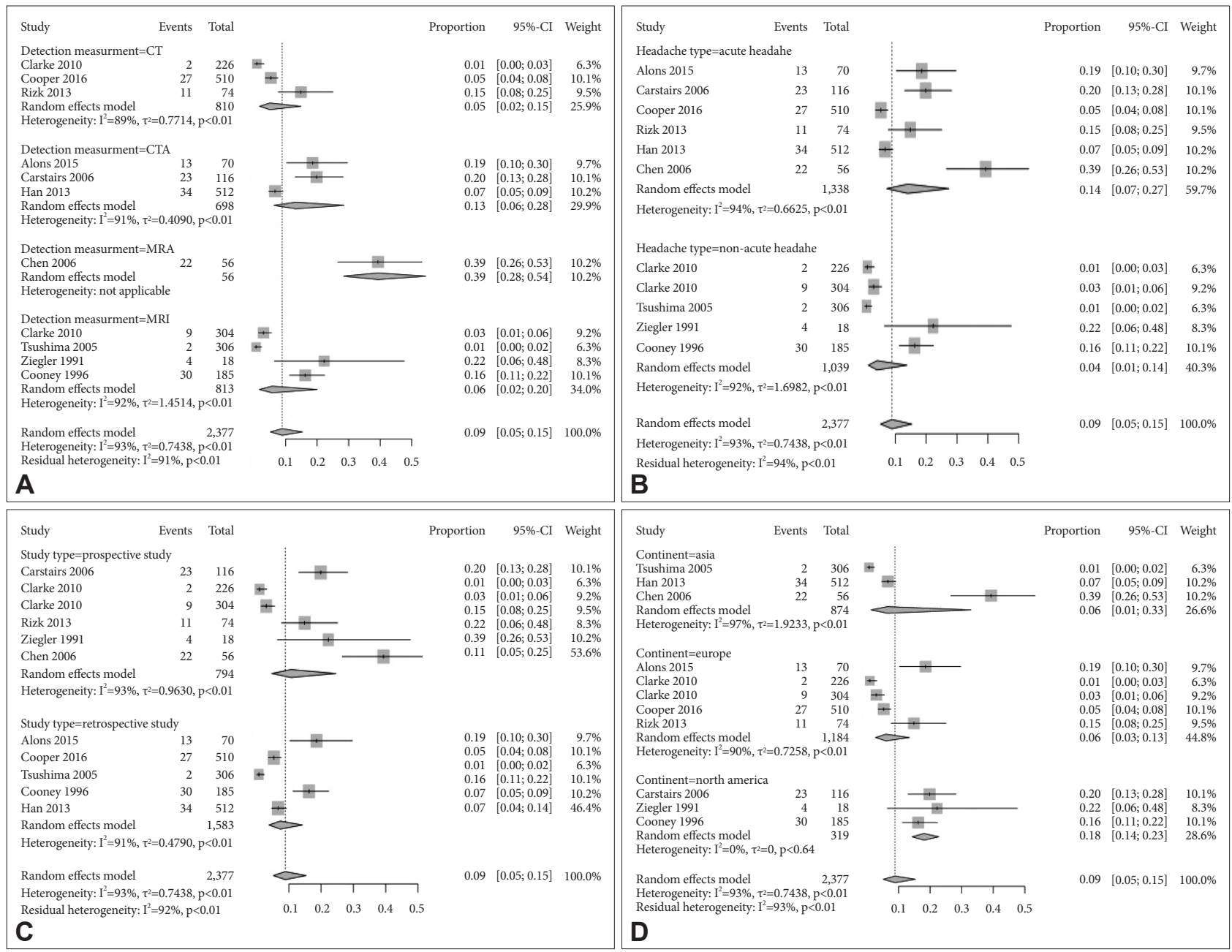

Figure 3. Forest plots for the subgroup analyses. Forest plot (A) showing the subgroup analysis by detection method. Forest plot (B) showing the subgroup analysis by headache type. Forest plot (C) showing the subgroup analysis by study type. Forest plot (D) showing the subgroup analysis by study region. 


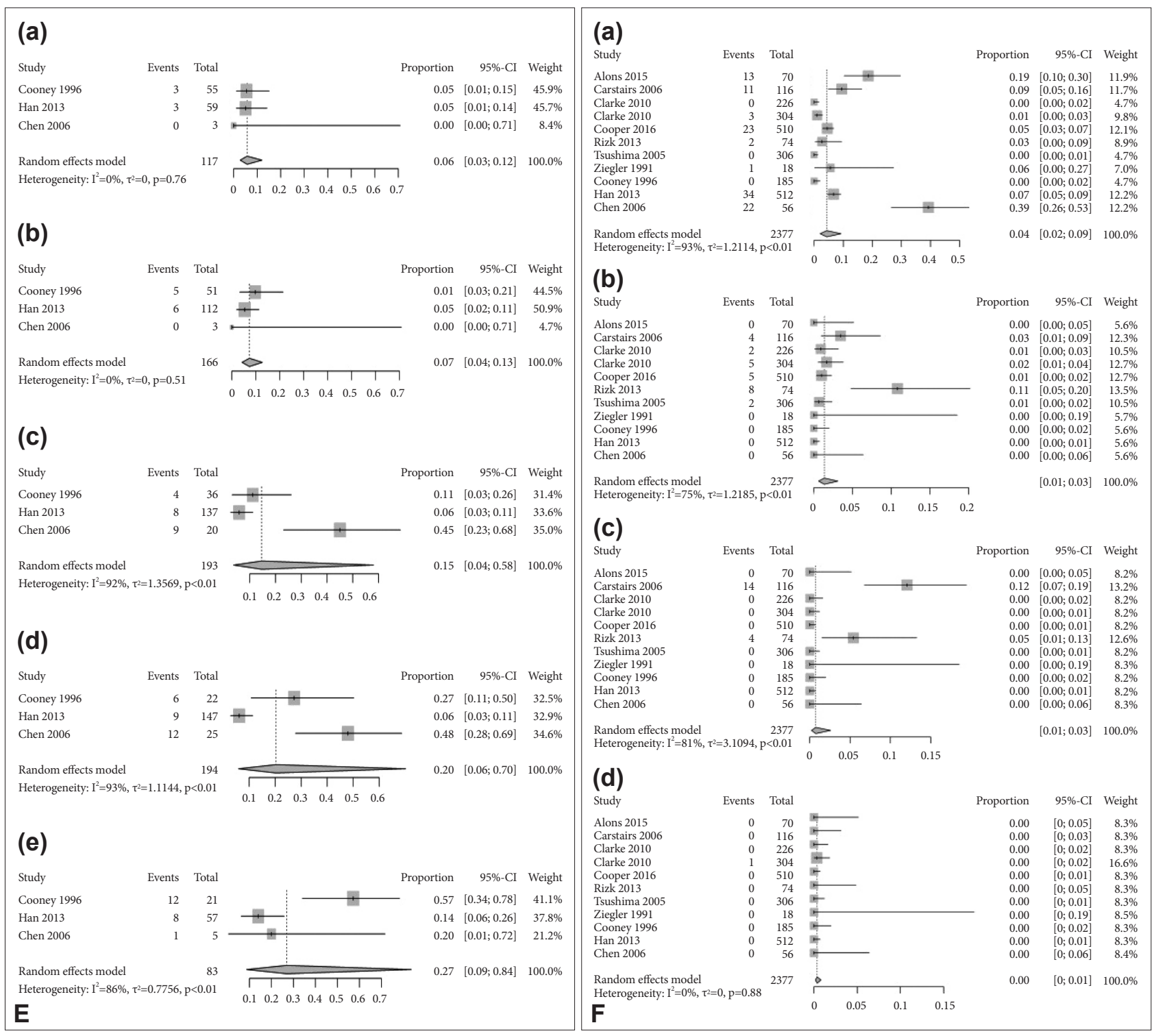

Figure 3. Forest plots for the subgroup analyses (continued). Forest plot (E) showing the subgroup analysis by age group. Forest plot $(F)$ showing the subgroup analysis by disease type. In forest plot $E$, each forest plot indicates the pooled incidence by age group; (a) is $\leq 30$, (b) is $31-40$, (c) is $41-50$, (d) is $51-60$, and (e) is $>61$. In forest plot $F$, each plot indicates the pooled prevalence that each disease will be found. (a) A kind of cerebrovascular disease, (b) a brain space-occupying lesions, (c) an infectious/inflammatory disease, and (d) congenital human brain malformations.

\section{DISCUSSION}

We performed a systematic literature review and metaanalysis to examine the prevalence of detecting clinically significant pathologies via neuroimaging in patients with suspected primary headache. The present findings are important because this study included patients with various types of headache, such as migraine, tension type headache, acute severe headache, and chronic/recurrent headache, to identify the value of using neuroimaging as an initial diagnostic tool. Furthermore, diverse subgroup meta-analyses based on detection method, headache type, study type, study region, age group, and disease type were performed to examine these factors as sources of potential heterogeneity and to provide clinically useful information. The present analyses found a $8.86 \%$ prevalence of detecting clinically significant lesions in primary headache patients, which is consistent with previous studies. Clinically significant abnormalities and/or pathologies, including brain cancer, tumors, deformities, hemorrhages, and infarctions, can be a cause of headache. A previous meta-analysis of 19 studies involving adult patients presenting with headache to an emergency department who underwent non-contrast CT head scans reported detection rates of $6.7 \%$ (95\% CI: 5.9-7.5\%) in non-traumatic headache patients who 
underwent a normal neurological exam and $8 \%$ (95\% CI: $7.3-8.7 \%$ ) in patients with altered mental status or any focal neurological findings. ${ }^{6}$ In another systematic review, the prevalence of significant intracranial abnormalities in migraine headache patients ranged from $0 \%$ to $3.1 \%$ among 11 studies, and the probability of a headache abnormality being classified as "unspecified" was $0-6.7 \% .{ }^{16}$ A meta-analysis of the use of MRI to detect chronic or recurrent headaches estimated an overall detection rate of $2.1 \%$ (upper $99.5 \%$ confidence bound: $3.4 \%$ ) in unspecified headache patients and $0.52 \%$ in migraine patients without any other neurological findings. ${ }^{17}$ Clarke et al. $^{26}$ found that the prevalence of detecting significant abnormalities by neuroimaging increased from $1.2 \%$ to $5.5 \%$ when an intracranial abnormality or other high-risk pathology was the provisional diagnosis. Although the studies involved various types of headache and brain imaging techniques, the prevalence of detecting a clinically significant abnormality on neuroimaging was usually below $10 \%$. A common recommendation in all review articles was judicious and selective use of neuroimaging in headache patients with abnormal neurological findings or atypical symptoms on physical examination.

In the present study, various subgroup meta-analyses based on neuroimaging tools, headache type, study type, continent, age group, and disease type were conducted. Performing subgroup meta-analyses and systematic reviews on various neuroimaging tools allows us to consider the respective advantages and disadvantages of each imaging modality. The prevalences of each neuroimaging technique cannot be compared directly, since selection bias can induce differences in the rate of detecting structural abnormalities. This bias occurs because of the different types of neuroimaging methods used depending on the headache type and patient symptoms. According to a national clinical guideline, CT should be performed in patients with thunderclap headache when SAH is suspected whereas MRI should be considered in those with cluster headache, paroxysmal hemicranias, or short-lasting unilateral neuralgiform headache attacks with conjunctival injection and tearing (SUNCT). ${ }^{12}$ SUNCT is rare headache disorder characterized by extremely frequent attacks of unilateral head pain and autonomic activation that is considered to be a trigeminal autonomic cephalgia. ${ }^{29}$ In addition to the conventional uses of CT and MRI, CTA and MRA are becoming increasingly recognized as feasible tools for the detection of intracranial abnormalities in headache patients. Previous studies have shown that CTA is highly sensitive for detecting aneurysms in patients with thunderclap headaches and that MRA is useful for detecting small intracranial aneurysms $(3-4 \mathrm{~mm}) .^{25,30}$ However, CT and CTA carry some risks, including potential adverse reactions to the contrast medium (e.g., allergic reac- tion and renal dysfunction) and radiation exposure.10 The disadvantages of MRI and MRA include the high cost of operation, long image acquisition time, limited access, and the need for greater technical expertise to interpret the obtained images compared to CT scans. ${ }^{10,24}$ Given these respective advantages and disadvantages, guidelines regarding the selection of appropriate neuroimaging tools require further evaluation and studies.

Previous studies have distinguished between acute severe headaches (e.g., thunderclap headache) and non-acute headaches (e.g., migraine, tension-type headache). The former is a severe headache that peaks within a few minutes and lasts more than an hour. It can be a symptom of secondary headache syndromes such as SAH and cerebral venous thrombosis.9 In the present study, the prevalence of detecting significant neurological abnormalities was slightly higher in acute [13.98\% (95\% CI: 7.14-27.39\%)] than non-acute [4.06\% (95\% CI: $1.20-13.77 \%)]$ headache patients, similar to previous studies. In a previous meta-analysis, vascular abnormalities were identified by CTA in $7.4 \%$ of patients with acute severe headache and normal neurological examination results. ${ }^{31} \mathrm{~A}$ large prospective multicenter study detected SAH in $7.7 \%$ of 3,132 patients with acute headache. ${ }^{32}$ Another meta-analysis identified significant abnormalities using CT or MRI in approximately $0.2 \%$ of non-acute headache patients with normal neurological examination results. ${ }^{16}$ In a large prospective consecutive study of 1,876 patients with non-acute headaches, significant intracranial lesions were found in 22 of the patients (1.2\%), and the prevalence of clinically significant abnormalities in headache patients with normal neurological examination results was $0.9 \%{ }^{2}$ These results suggest that neuroimaging can detect abnormal findings at a higher rate in acute headache patients than in non-acute headache patients. In comparisons of study type, there was little difference in prevalence between prospective [10.64\% (95\% CI: 4.60-24.63)] and retrospective [7.31\% (95\% CI: 3.77-14.18)] studies. Retrospective studies are associated with a high risk of bias and are limited by data collection; however, those limitations appear to have little effect on the detection of abnormal findings by neuroimaging. The prevalence was also not affected by study region. The prevalence was slightly higher in North America [18.03\% (95\% CI: 14.26-22.81\%)] than in other continents [Asia: 6.43\% (95\% CI: 1.25-32.95\%); Europe: 5.98\% (95\% CI: 2.66$13.46 \%)$, which may reflect differences in evaluation methods by ethnicity, insurance status, and guidelines across continents or countries. In a systematic analysis of the global, regional, and national burden of neurological disorders, the prevalence and disability-adjusted life years of the different types of headache exhibited a three- to four-fold variation; ${ }^{33}$ however, further research is needed to determine the prevalence ac- 
cording to study type and region.

A previous set of guidelines recommended neuroimaging for patients older than 50 years of age with new-onset headache disorder. ${ }^{11}$ Additionally, Chen et al. ${ }^{28}$ reported that vasoconstriction is more common in middle-aged patients and those with atypical headaches, which are more frequent or severe than a typical migraine. Among the 10 studies selected for the present meta-analysis, detailed age information was available in three studies. ${ }^{23,24,28}$ Therefore, patient age was divided into five categories for the subgroup meta-analysis: $\leq 30,30$ s, 40 s, 50 s, and $>60$ years of age. The prevalence rates of clinically significant intracranial abnormalities were $5.73 \%$ in the $\leq 30$ group (95\% CI: $2.72-12.07 \%$ ), $7.34 \%$ in the 30 s group (95\% CI: $4.21-12.78 \%$ ), $14.60 \%$ in the 40 s group (95\% CI: $3.67-58.03 \%$ ), $20.29 \%$ in the 50 s group (95\% CI: $5.86-70.20 \%$ ), and $26.93 \%$ in the $>60$ s group (95\% CI: $8.69-$ $83.52 \%)$. Taken together, these results suggest that there is a greater probability of identifying an intracranial disease in older patients. More specifically, the discovery rate of abnormalities more than doubled in patients in their $40 \mathrm{~s}$ and older. However, additional research will be needed to confirm this result due to the high between-study heterogeneity in patients above their $40 \mathrm{~s}$.

Finally, a subgroup meta-analysis was conducted according to disease type to determine which intracranial pathology could be expected when planning neuroimaging for patients with complaints of subjective headache. The identified intracranial diseases were categorized into four major groups, and it was revealed that the prevalence of finding cerebrovascular disease was highest [4.31\% (95\% CI: 2.01-9.24\%)], followed by brain space-occupying lesions [1.35\% (95\% CI: 0.59$3.11 \%)]$, infectious/inflammatory disease [0.72\% (95\% CI: $0.20-2.57 \%)]$, and congenital human brain malformations $[0.36 \%$ (95\% CI: 0.16-0.81\%)]. Thus, cerebrovascular diseases, such as SAH, infarcts, and cerebral thrombosis, were the most likely to be detected. The signs and symptoms associated with SAH include sudden onset thunderclap headache, neck stiffness, nausea, vomiting, photophobia, a decreased level of consciousness, syncope, focal neurological deficits, seizure, drowsiness, confusion, and agitation. ${ }^{34,35}$ However, previous studies have shown that some of these symptoms overlap with those of sub-acute carbon monoxide poisoning, including headaches, nausea, vomiting, dizziness, muscular weakness, and blurred vision. ${ }^{36}$ When performing neuroimaging for headache patients complaining of these symptoms, one might expect to identify cerebrovascular disease.

There are limitations to the present study. First, only one study using MRA to diagnose headache was assessed, and the number of subjects in that study was not sufficient. Thus, more studies are needed to compare the utility of MRA with other neuroimaging tools. Second, our literature search included only published articles, and the underlying selection bias in published articles may have affected the prevalence rates more so than in previous meta-analyses. Third, the heterogeneity of the study made it difficult to obtain valid and consistent results despite the use of standardized analytical methods. Future research is needed to compensate for these limitations, especially in terms of standardization of neuroimaging tools. Currently, CT, CTA, MRI, and MRA are used for neuroimaging in headache patients, and standardized guidelines for effective use of each modality are needed. The unification of terms associated with neuroimaging diagnoses would also be of great benefit. In headache patients diagnosed by neuroimaging tools, the diagnoses or disease names are often heterogeneous, which may ultimately lead to a decreased standard of care. The differences in diagnoses of headache patients according to various medical environments, including primary care, secondary care, tertiary care, emergency room, and outpatient care, should also be evaluated. Finally, the cost-effectiveness of neuroimaging in headache patients needs to be determined. Until now, neuroimaging has been considered unnecessary for primary headache patients, especially those with chronic headache and no focal neurological signs. ${ }^{5,6,37}$ As such, cost effectiveness should be evaluated according to headache type, symptoms and signs, and the implemented neuroimaging tool.

The present study conducted a systematic literature review and meta-analysis that focused on neuroimaging for headache patients. Because the overall prevalence of a clinically significant intracranial pathology in headache patients who underwent neuroimaging was $8.86 \%$, neuroimaging methods should be utilized for headache patients in a careful and limited manner due to the occurrence of various side effects and to reduce unnecessary imaging. In the case of acute headache patients who visit an emergency department, angiography tests, such as CTA or MRA, may be useful for identifying underlying vascular abnormalities, but this issue will require further research to reach a definite conclusion. Additionally, further research will be needed regarding differences in the prevalence of headaches according to race, age, and work-up due to health care systems and insurance. In conclusion, this study revealed significant implications for meta-analyses of various headache patients and neuroimaging tools. Future studies in this area will help in the diagnosis of primary headaches by neuroimaging.

\section{Supplementary Materials}

The online-only Data Supplement is available with this article at https://doi.org/10.30773/pi.2019.04.11. 


\section{Acknowledgments}

This research was supported by a grant of the Korea Health Technology R\&D Project through the Korea Health Industry Development Institute (KHIDI), funded by the Ministry of Health \& Welfare, Republic of Korea (grant number: $\mathrm{HC17C0117).}$

\section{Conflicts of Interest}

The authors have no potential conflicts of interest to disclose.

\section{Author Contributions}

Conceptualization: Hye Youn Park, Sun Mi Kim. Data curation: Ye Eun Jang, Hee Yea Choi. Formal analysis: Eun Young Cho. Funding acquisition: Hye Youn Park. Investigation: Ye Eun Jang, Hye Youn Park, Hee Yea Choi. Methodology: Ye Eun Jang, Eun Young Cho. Project administration: Hye Youn Park. Resources: Ye Eun Jang, Hye Youn Park, Hee Yea Choi. Software: Ye Eun Jang, Hye Youn Park. Supervision: Hye Youn Park, Sun Mi Kim. Validation: Hye Youn Park, Sun Mi Kim. Visualization: Ye Eun Jang, Eun Young Cho. Writing_-original draft: Ye Eun Jang, Hye Youn Park, Eun Young Cho. Writing_-review \& editing: Ye Eun Jang, Hye Youn Park.

\section{ORCID iDs}

Hye Youn Park https://orcid.org/0000-0002-5097-532X

Ye Eun Jang https://orcid.org/0000-0001-6461-9986

\section{REFERENCES}

1. Headache Classification Committee of the International Headache Society (IHS). The International Classification of Headache Disorders, 3rd edition. Cephalalgia 2018;38:1-211.

2. Sandrini G, Friberg L, Coppola G, Jänig W, Jensen R, Kruit M, et al. Neurophysiological tests and neuroimaging procedures in non-acute headache (2nd edition). Eur J Neurol 2011;18:373-381.

3. National Clinical Guideline Centre. Headaches: Diagnosis and Management of Headaches in Young People and Adults. London (UK): National Institute for Health and Clinical Excellence (NICE); 2012 Sep. 38p. (Clinical Guideline; no.150).

4. Headache Classification Subcommittee of the International Headache Society. The International Classification of Headache Disorders: 2nd edition. Cephalalgia 2004;24(Suppl 1):9-160.

5. Lepage R, Krebs L, Kirkland S, Alexiu C, Campbell S, Rowe B. MP25: the role of advanced imaging in the management of benign headaches in the emergency department: a systematic review. CJEM 2017;19:S73.

6. Williams A, Friedman BW. The yield of non-contrast cranial computed tomography for the detection of intracranial pathology in emergency department patients with headache: a systematic review. Ann Emerg Med 2014;64:S48.

7. Vernooij MW, Ikram MA, Tanghe HL, Vincent AJ, Hofman A, Krestin GP, et al. Incidental findings on brain MRI in the general population. N Engl J Med 2007;357:1821-1828.

8. Weber F, Knopf H. Incidental findings in magnetic resonance imaging of the brains of healthy young men. J Neurol Sci 2006;240:81-84.

9. Alons IM, van den Wijngaard IR, Verheul RJ, Lycklama à Nijeholt G, Wermer MJ, Algra A, et al. The value of CT angiography in patients with acute severe headache. Acta Neurol Scand 2015;131:164-168.

10. Rizk B, Platon A, Tasu JP, Becker CD, Mendes Pereira V, Perneger T, et al. The role of unenhanced CT alone for the management of headache in an emergency department. A feasibility study. J Neuroradiol 2013; 40:335-341.

11. Beithon J, Gallenberg M, Johnson K, Kildahl P, Krenik J, Liebow M, et al. Diagnosis and Treatment of Headache. Bloomington (MN): Institute for Clinical Systems Improvement (ICSI); 2013.

12. Scottish Intercollegiate Guidelines Network. Diagnosis and Management of Headache in Adults. Edinburgh: NHS Quality Improvement Scotland; 2008.
13. May A. New insights into headache: an update on functional and structural imaging findings. Nat Rev Neurol 2009;5:199-209.

14. Detsky ME, McDonald DR, Baerlocher MO, Tomlinson GA, McCrory DC, Booth CM. Does this patient with headache have a migraine or need neuroimaging? JAMA 2006;296:1274-1283.

15. Halker R, Vargas B, Dodick DW. Cluster headache: diagnosis and treatment. Semin Neurol 2010;30:175-185.

16. Frishberg BM, Rosenberg JH, Matchar DB, McCrory DC, Pietrzak MP, Rosen TD, et al. Evidence-Based Guidelines in the Primary Care Setting: Neuroimaging in Patients with Nonacute Headache. St Paul, MN: US Headache Consortium; 2000.

17. Tsushima Y, Endo K. MR imaging in the evaluation of chronic or recurrent headache. Radiology 2005;235:575-579.

18. Kim SY, Park JE, Seo HJ, Lee YJ, Jang BH, Son HJ, et al. NECA's Guidance for Undertaking Systematic Reviews and Meta-Analyses for Intervention. Seoul: National Evidence-based Healthcare Collaborating Agency; 2011.

19. Hoy D, Brooks P, Woolf A, Blyth F, March L, Bain C, et al. Assessing risk of bias in prevalence studies: modification of an existing tool and evidence of interrater agreement. J Clin Epidemiol 2012;65:934-939.

20. Higgins J, Green S. Cochrane handbook for systematic reviews of interventions Version 5.1. 0. The Cochrane Collaboration. Confidence Intervals; 2011.

21. Hwang SD. Meta-Analysis Using R. Seoul: Hakgissa; 2015.

22. Cooper JG, Smith B, Hassan TB. A retrospective review of sudden onset severe headache and subarachnoid haemorrhage on the clinical decision unit: looking for a needle in a haystack? Eur J Emerg Med 2016;23:356-362.

23. Cooney BS, Grossman RI, Farber RE, Goin JE, Galetta SL. Frequency of magnetic resonance imaging abnormalities in patients with migraine. Headache 1996;36:616-621.

24. Han A, Yoon DY, Kim ES, Kim HC, Cho BM, Lee JY. Value of CT angiography for the detection of intracranial vascular lesions in patients with acute severe headache. Eur Radiol 2013;23:1443-1449.

25. Carstairs SD, Tanen DA, Duncan TD, Nordling OB, Wanebo JE, Paluska TR, et al. Computed tomographic angiography for the evaluation of aneurysmal subarachnoid hemorrhage. Acad Emerg Med 2006;13:486-492.

26. Clarke CE, Edwards J, Nicholl DJ, Sivaguru A. Imaging results in a consecutive series of 530 new patients in the Birmingham Headache Service. J Neurol 2010;257:1274-1278.

27. Ziegler DK, Batnitzky S, Barter R, McMillan JH. Magnetic resonance image abnormality in migraine with aura. Cephalalgia 1991;11:147-150.

28. Chen SP, Fuh JL, Lirng JF, Chang FC, Wang SJ. Recurrent primary thunderclap headache and benign CNS angiopathy: spectra of the same disorder? Neurology 2006;67:2164-2169.

29. Cohen AS, Matharu MS, Goadsby PJ. Short-lasting unilateral neuralgiform headache attacks with conjunctival injection and tearing (SUNCT) or cranial autonomic features (SUNA)-a prospective clinical study of SUNCT and SUNA. Brain 2006;129:2746-2760.

30. Ross JS, Masaryk TJ, Modic MT, Ruggieri PM, Haacke EM, Selman WR. Intracranial aneurysms: evaluation by MR angiography. AJR Am J Roentgenol 1990;155:159-165.

31. Alons IME, Goudsmit BFJ, Jellema K, van Walderveen MAA, Wermer $\mathrm{MJH}$, Algra A. Yield of computed tomography (CT) angiography in patients with acute headache, normal neurological examination, and normal non contrast CT: a meta-analysis. J Stroke Cerebrovasc Dis 2018;27:1077-1084.

32. Perry JJ, Stiell IG, Sivilotti ML, Bullard MJ, Emond M, Symington C, et al. Sensitivity of computed tomography performed within six hours of onset of headache for diagnosis of subarachnoid haemorrhage: prospective cohort study. BMJ 2011;343:d4277.

33. GBD 2015 Neurological Disorders Collaborator Group. Global, regional, and national burden of neurological disorders during 19902015: a systematic analysis for the Global Burden of Disease Study 
2015. Lancet Neurol 2017;16:877-897.

34. Cortelli P, Cevoli S, Nonino F, Baronciani D, Magrini N, Re G, et al. Evidence-based diagnosis of nontraumatic headache in the emergency department: a consensus statement on four clinical scenarios. Headache 2004;44:587-595.

35. Bethel J, McInulty L. Subarachnoid haemorrhage: case study and literature review. Emerg Nurse 2010;18:22-26.
36. Steiner T, MacGregor E, Davies P. Guidelines for All Healthcare Professionals in the Diagnosis and Management of Migraine, TensionType, Cluster and Medication-Overuse Headache. 3rd Edition. Hull: British Association for the Study of Headache; 2007.

37. Kuruvilla DE, Lipton RB. Appropriate use of neuroimaging in headache. Curr Pain Headache Rep 2015;19:17. 


\begin{tabular}{|c|c|c|c|c|}
\hline \multicolumn{5}{|c|}{ Pubmed Search } \\
\hline & & Key word & Result & Date \\
\hline & $\# 1$ & $\begin{array}{l}\text { Headache* }[\text { MeSHTerms }] \text { OR ClusterHeadache* }[\text { MeSHTerms }] \text { OR } \\
\text { MigraineDisorder*[MeSHTerms }] \text { OR Tension-Type Headache*[MeSH Terms }] \text { OR } \\
\text { Vascular Headache* }[\text { MeSH Terms }]\end{array}$ & 50,147 & $2018-01-25$ \\
\hline & $\# 2$ & $\begin{array}{l}\text { Headache*[Title/Abstract] OR Cluster Headache*[Title/Abstract] OR } \\
\text { Migraine*[Title/Abstract] OR Tension-Type Headache*[Title/Abstract] OR } \\
\text { Tension Type Headache*[Title/Abstract] OR Vascular Headache*[Title/Abstract] }\end{array}$ & 89,782 & 2018-01-25 \\
\hline \multirow[t]{3}{*}{$\mathrm{P}$} & $\# 3$ & $\# 1 \mathrm{OR} \# 2$ & 100,275 & $2018-01-25$ \\
\hline & \#4 & $\begin{array}{l}\text { Tomography, X-Ray Computed[MeSH Terms] OR Computed Tomography } \\
\text { Angiography*[MeSH Terms] OR Magnetic Resonance Imaging*[MeSH Terms] OR } \\
\text { Diffusion Magnetic Resonance Imaging*[MeSH Terms] OR Magnetic Resonance } \\
\text { Angiography*[MeSH Terms] OR Diagnostic Imaging*[MeSH Terms] }\end{array}$ & $2,395,285$ & 2018-01-25 \\
\hline & $\# 5$ & $\begin{array}{l}\text { CT[Title/Abstract] OR Computed Tomography Angiography*[Title/Abstract] OR } \\
\text { Magnetic Resonance Imaging*[Title/Abstract] OR MRI[Title/Abstract] OR } \\
\text { MRIs[Title/Abstract] OR Diffusion Magnetic Resonance Imaging*[Title/Abstract] OR } \\
\text { Magnetic Resonance Angiography*[Title/Abstract] OR Diagnostic Imaging*[Title/Abstract] }\end{array}$ & 566,668 & 2018-01-25 \\
\hline \multirow[t]{3}{*}{ I } & \#6 & $\# 4 \mathrm{OR} \# 5$ & $2,582,568$ & 2018-01-25 \\
\hline & $\# 7$ & $\begin{array}{l}\text { Evaluation Studies as Topic*[MeSH Terms] OR Evidence-Based Medicine*[MeSH Terms] } \\
\text { OR Practice Guidelines as Topic*[MeSH Terms] OR Guideline*[MeSH Terms] OR } \\
\text { Practice Guideline*[MeSH Terms] }\end{array}$ & $1,079,793$ & 2018-01-25 \\
\hline & $\# 8$ & $\begin{array}{l}\text { EvaluationStudiesasTopic*[TextWord]OREvidence-BasedMedicine*[TextWord]OR } \\
\text { Evidence Based Medicine*[Text Word] OR Practice Guidelines as Topic*[Text Word] OR } \\
\text { Guideline*[Text Word] OR Practice Guideline*[Text Word] }\end{array}$ & 554,666 & 2018-01-25 \\
\hline $\mathrm{O}$ & $\# 9$ & $\# 7 \mathrm{OR} \# 8$ & $1,340,670$ & 2018-01-25 \\
\hline Sum & $\# 10$ & \#3 AND \#6 AND \#9 & 564 & 2018-01-25 \\
\hline Limit & $\# 11$ & $\begin{array}{l}\text { adult }[\text { MeSH Terms] OR (adult*[Text Word] NOT adolescent*[Text Word] NOT } \\
\text { child*[Text Word] })\end{array}$ & $6,890,962$ & 2018-01-25 \\
\hline Final & $\# 12$ & \#10 AND \#11 & 354 & 2018-01-25 \\
\hline \multicolumn{5}{|c|}{ Embase Search } \\
\hline \multirow{5}{*}{$\mathrm{P}$} & $\# 1$ & $\begin{array}{l}\text { headache':ti,ab,kw OR 'cluster headache':ti,ab,kw OR ‘migraine’:ti,ab,kw OR } \\
\text { 'tension headache':ti,ab,kw OR 'vascular headache':ti,ab,kw OR 'migraine disorder':ti,ab,kw }\end{array}$ & 121,040 & $2018-01-25$ \\
\hline & $\# 2$ & $\begin{array}{l}\text { headache'/exp OR 'cluster headache'/exp OR 'migraine'/exp OR 'tension headache'/exp } \\
\text { OR 'vascular headache'/exp }\end{array}$ & 226,116 & 2018-01-25 \\
\hline & $\# 3$ & $\# 1 \mathrm{OR} \# 2$ & 248,388 & 2018-01-25 \\
\hline & $\# 4$ & $\begin{array}{l}\text { computed tomographic angiography':ti,ab,kw OR 'computed tomography scanner':ti,ab,kw } \\
\text { OR 'functional magnetic resonance imaging':ti,ab,kw OR 'nuclear magnetic resonance } \\
\text { imaging':ti,ab,kw OR 'diffusion weighted imaging':ti,ab,kwOR 'magnetic resonance } \\
\text { angiography':ti,ab,kw OR 'diagnostic imaging':ti,ab,kw OR 'ct':ti,ab,kw OR 'mri':ti,ab,kw }\end{array}$ & 790,680 & 2018-01-25 \\
\hline & \#5 & $\begin{array}{l}\text { computed tomographic angiography'/expOR 'computed tomography scanner'/exp } \\
\text { OR 'functional magnetic resonance imaging'/exp OR 'nuclear magnetic resonance imaging'/ } \\
\text { exp OR 'diffusion weighted imaging'/exp OR 'magnetic resonance angiography'/exp OR } \\
\text { 'diagnostic imaging'/exp }\end{array}$ & 926,794 & 2018-01-25 \\
\hline \multirow[t]{3}{*}{ I } & \#6 & $\# 4 \mathrm{OR} \# 5$ & $1,294,872$ & 2018-01-25 \\
\hline & $\# 7$ & $\begin{array}{l}\text { evaluation study’tti,ab,kw OR 'evidence based medicine’:ti,ab,kw OR 'practice guideline’:ti,ab,kw } \\
\text { OR 'clinicalpractice':ti,ab,kw OR 'guideline’:ti,ab,kw OR 'best practice':ti,ab,kw OR 'clinical } \\
\text { practice guideline':ti,ab,kw }\end{array}$ & 301,610 & 2018-01-25 \\
\hline & $\# 8$ & $\begin{array}{l}\text { evaluation study'/exp OR ‘evidence based medicine'/exp OR 'practice guideline'/exp } \\
\text { OR 'clinical practice'/exp OR 'guideline/exp }\end{array}$ & $1,666,838$ & 2018-01-25 \\
\hline $\mathrm{O}$ & $\# 9$ & $\# 7 \mathrm{OR} \# 8$ & $1,798,386$ & 2018-01-25 \\
\hline Sum & $\# 10$ & \#3 AND \#6 AND \#9 & 2,169 & 2018-01-25 \\
\hline Limit & $\# 11$ & adult':ti,ab,kw OR 'adult'/exp & $7,311,520$ & $2018-01-25$ \\
\hline Final & $\# 12$ & \#10 AND \#11 & 1,131 & 2018-01-25 \\
\hline
\end{tabular}

